\title{
Carbon and Oxygen in the Spectrum of HR 8799c
}

\author{
Q. M. Konopacky ${ }^{1,5}$, T. S. Barman ${ }^{2}$, B. A. Macintosh ${ }^{3}$ and C. Marois ${ }^{4}$ \\ ${ }^{1}$ Dunlap Institute for Astronomy and Astrophysics, University of Toronto, ${ }^{2}$ Lowell \\ Observatory, ${ }^{3}$ Larence Livermore National Laboratory, ${ }^{4}$ National Research Council Canada, \\ Dominion Astrophysical Observatory \\ ${ }^{5}$ Dunlap Fellow
}

\begin{abstract}
The field of exoplanet spectroscopy has grown tremendously in the last decade. With the discovery of gas giant planets at wide separations from their host stars via direct imaging, it is now possible to obtain exoplanet spectra with unprecedented spectral resolution. We present a medium resolution spectrum of the directly imaged exoplanet HR 8799c. This $K$-band spectrum was obtained using the integral field spectrograph OSIRIS on the Keck II telescope. Our spectrum shows numerous, well-resolved molecular lines from water and carbon monoxide $(\mathrm{CO})$. There is no clear evidence for methane absorption, in spite of a best fit temperature of $\sim 1100 \mathrm{~K}$. We find a best fit surface gravity $\log (g) \sim 4.0$, consistent with the inferred young age for the system ( $\sim 30 \mathrm{Myr})$, and a continuum morphology consistent with previously-inferred dust clouds. Using the water and $\mathrm{CO}$ lines, we are able to estimate the $\mathrm{C} / \mathrm{O}$ ratio for this planet. We find a ratio slightly higher than stellar $(\sim 0.65)$, which provides hints about the planet's formation.
\end{abstract}

Keywords. planetary systems: formation, techniques: spectroscopic

\section{Introduction}

The discovery of extrasolar planets through direct imaging has opened up a new laboratory for the characterization of exoplanets. By detecting the light from the planet itself, it is possible to probe the atmospheres of gas giant planets with spectroscopy. The four directly imaged planets around the young ( $30 \mathrm{Myr}$, Zuckerman et al. 2011) A type star HR 8799 are ideal for such a study (Marois et al. 2008, 2010). Previous studies have suggested that all four planets are between $\sim 5-10 \mathrm{M}_{J u p}$ and $\sim 900-1200 \mathrm{~K}$ (e.g., Marois et al. 2010, Marley et al. 2012). Their contrast at infrared wavelengths is sufficient that the planets are observable with current adaptive optics systems and integral field spectrographs on 8-10 meter telescopes. Here we present spectroscopic observations of the 3rd closest planet to the star, HR 8799c.

\section{Data and Analysis}

Data were obtained using the integral field spectrograph OSIRIS on the W.M. Keck II 10-meter telescope in 2010 and 2011. These observations were made in conjunction with the facility adaptive optics system in the $K$-band $(1.965 \mu \mathrm{m}$ to $2.381 \mu \mathrm{m})$. A total of 5.5 hours of observations were obtained. We used the speckle modeling and subtraction algorithms detailed in Barman et al. (2011). Figure 1 shows the final extracted spectrum at the full resolution of OSIRIS $(\mathrm{R} \sim 4000)$.

We then used theoretical atmosphere models to estimate properties of the HR $8799 \mathrm{c}$ and determine which molecules are present in its spectrum. The model atmosphere grid is described in Barman et al. (2011). We find copious $\mathrm{CO}$ and $\mathrm{H}_{2} \mathrm{O}$ lines in the spectrum. Conversely, there is no statistically significant detection of $\mathrm{CH}_{4}$. We also find a best-fit 


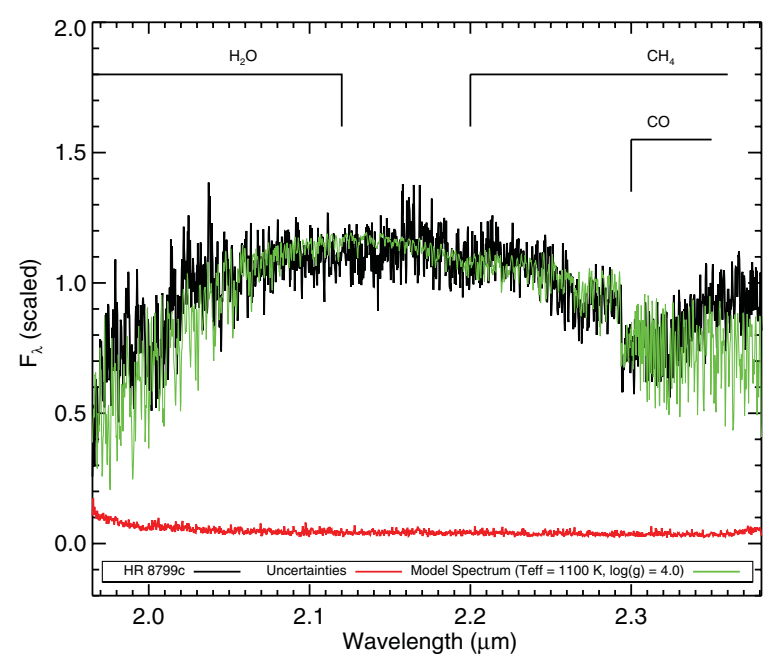

Figure 1. Extracted spectrum of HR $8799 \mathrm{c}$ (R 4000, black). Important opacity sources in $K$-band $\left(\mathrm{H}_{2} \mathrm{O}, \mathrm{CO}\right.$, and $\left.\mathrm{CH}_{4}\right)$ are highlighted. A best-fit PHOENIX atmosphere model template is overplotted in green. The uncertainties at each wavelength are plotted underneath in red for clarity (from Konopacky et al. 2013).

temperature of $1100 \pm 100 \mathrm{~K}$ and surface gravity $(\log (\mathrm{g}))$ of $3.0-4.0$. The best-fitting model is overplotted in Figure 1 in green. As in the case of HR 8799 b (e.g., Barman et al. 2011, Marley et al. 2012), we find that both clouds and non-equilibrium chemistry are necessary to reproduce the spectrum. The non-equilibrium chemistry allows for the presence of $\mathrm{CO}$ rather than $\mathrm{CH}_{4}$ at temperatures as low as $1100 \mathrm{~K}$, where in more massive field-type objects $\mathrm{CH}_{4}$ is a more dominant opacity source (e.g., Kirkpatrick 2005).

With our detections of $\mathrm{CO}$ and $\mathrm{H}_{2} \mathrm{O}$, we can begin to assess the ratio of carbon to oxygen in this planet. This ratio has been suggested as a possible tracer of formation mechanism, where high $\mathrm{C} / \mathrm{O}$ ratio may signal formation via core accretion while a stellar $\mathrm{C} / \mathrm{O}$ ratio implies formation via gravitational instability (Öberg et al. 2011). We generated a grid of models with $\mathrm{C}$ and $\mathrm{O}$ abundances varying based on a simple model of solid condensation in a protoplanetary disk around an A-type star (Öberg et al. 2011) and fit this grid to our spectrum. We find a best-fit $\mathrm{C} / \mathrm{O}$ ratio of $0.65_{-0.05}^{+0.10}$, slightly elevated from the assumed $\mathrm{C} / \mathrm{O}$ ratio of $\mathrm{HR} 8799 \mathrm{~A}$ of $\sim 0.55$. This is consistent with a core accretion formation scenario provided that the primarily gas atmosphere accreted a small amount of solids during formation. However, the uncertainties in the abundances of the star and other model considerations do not allow for the exclusion of either formation scenario.

A more detailed description of this work can be found in Konopacky et al. (2013).

\section{References}

Barman, T. S., Macintosh, B., Konopacky, Q. M., \& Marois, C. 2011, ApJ, 733, 65

Kirkpatrick, J. D. 2005, ARA\&A, 43, 195

Konopacky, Q. M., Barman, T. S., Macintosh, B. A., \& Marois, C. 2013, Science, 339, 1398

Marley, M. S., Saumon, D., Cushing, M., et al. 2012, ApJ, 754, 135

Marois, C., Macintosh, B., Barman, T., et al. 2008, Science, 322, 1348

Marois, C., Zuckerman, B., Konopacky, Q. M., Macintosh, B., \& Barman, T. 2010, Nature, 468, 1080

Öberg, K. I., Murray-Clay, R., \& Bergin, E. A. 2011, ApJL, 743, L16

Zuckerman, B., Rhee, J. H., Song, I., \& Bessell, M. S. 2011, ApJ, 732, 61 